\title{
A Novel Rear-End Collision Detection Algorithm Based on GNSS Fusion and ANFIS
}

\author{
Rui Sun, ${ }^{1,2}$ Fei Xie,, ${ }^{3,4}$ Dabin Xue, ${ }^{1}$ Yucheng Zhang, ${ }^{1}$ and Washington Yotto Ochieng ${ }^{5}$ \\ ${ }^{1}$ College of Civil Aviation, Nanjing University of Aeronautics \& Astronautics, Nanjing 211106, China \\ ${ }^{2}$ State Key Laboratory of Geo-Information Engineering, Xian 710054, China \\ ${ }^{3}$ School of Electrical and Automation Engineering, Nanjing Normal University, Nanjing 210042, China \\ ${ }^{4}$ Jiangsu Key Laboratory of 3D Printing Equipment and Manufacturing, Nanjing Normal University, Nanjing 210042, China \\ ${ }^{5}$ Centre for Transport Studies, Imperial College London, London SW7 2AZ, UK
}

Correspondence should be addressed to Fei Xie; xiefei@njnu.edu.cn

Received 16 May 2017; Revised 16 October 2017; Accepted 23 October 2017; Published 21 November 2017

Academic Editor: Richard S. Tay

Copyright (c) 2017 Rui Sun et al. This is an open access article distributed under the Creative Commons Attribution License, which permits unrestricted use, distribution, and reproduction in any medium, provided the original work is properly cited.

\begin{abstract}
Rear-end collisions are one of the most common types of accidents on roads. Global Satellite Navigation Systems (GNSS) have recently become sufficiently flexible and cost-effective in order to have great potential for use in rear-end collision avoidance systems (CAS). Nevertheless, there are two main issues associated with current vehicle rear-end CAS: (1) achieving relative vehicle positioning and dynamic parameters with sufficiently high accuracy and (2) a reliable method to extract the car-following status from such information. This paper introduces a novel integrated algorithm for rear-end collision detection. Access to high accuracy positioning is enabled by GNSS, electronic compass, and lane information fusion with Cubature Kalman Filter (CKF). The judgment of the car-following status is based on the application of the Adaptive Neurofuzzy Inference System (ANFIS). The field test results show that the designed algorithm could effectively detect rear-end collisions with an accuracy of $99.61 \%$ and a false alarm rate of $5.26 \%$ in the $10 \mathrm{~Hz}$ output rate.
\end{abstract}

\section{Introduction}

Rear-end collisions are a common type of traffic accident in which a vehicle crashes into the vehicle in front of it. According to statistics from the National Highway Traffic Safety Administration (NHTSA), this type of accident accounts for about one-third of all traffic accidents [1]. 30\% of rear-end collisions lead to injuries, and even though only $1 \%$ result in fatalities, the prevalence of this type of accident means that the social and economic costs are significant, such as property loss and traffic congestion [2]. In general, rear-end collisions are caused by human errors in the longitudinal driving task called car following, in which the driver fails to maintain a proper speed and safe distance from the vehicle in front. If appropriate measures could be taken in advance, for example, if an early warning could be provided, the probability of a collision could be greatly reduced. Intelligent Transport Systems (ITS) technologies which use advanced sensors and communication technologies for the assessment of real-time car-following status would thus be a beneficial addition to collision avoidance systems.

Various methods and algorithms related to rear-end collision avoidance have been described in the literature in recent years. Araki et al. [3,4] developed an onboard laser radar and a Charge Coupled Device (CCD) camera integrated system with fuzzy logic to evaluate the potential collision status. Tsai [5] also presented a laser radar based vehicle safety and warning system, while Ueki et al. [6] developed a vehicular collision avoidance system by means of intervehicle communication technology. Huang and Tan [7] discussed the engineering feasibility of a cooperative collision warning system based on a future trajectory prediction algorithm for vehicles equipped with a Differential Global Positioning System (DGPS) unit and other related motion sensors. Ong and Lachapelle [8] proposed a GNSS based vehicle-pedestrian and vehicle-cyclist crash avoidance system, analysing the performance of GNSS in such a collision avoidance system, and Toledo-Moreo and Zamora-Izquierdo [9] developed an 
integrated lateral and longitudinal information-supported collision avoidance system integrated via GPS/IMU/digital maps. Milanés et al. [10] proposed a fuzzy logic based warning and braking system. Ujjainiya and Chakravarthi [11] proposed a cost-effective vehicle collision avoidance system based on vision sensors and image processing algorithms, and Alpar and Stojic [12] designed an intelligent rear-end collision warning algorithm based on license plate segmentation and a fuzzy logic based warning system.

Although the aforementioned rear-end collision avoidance detection approaches have shown some potential in certain conditions, several technical barriers remain to be surmounted. First, most of the research uses vision sensors, which are weather sensitive and thus not adaptive for wide applications. In addition, some research has adopted technologies such as scanning radar to provide relative positioning for the collision avoidance system, but in these cases the system performance is highly related to the cost of the sensors. Compared with the other technologies, GNSS and its fusion with other data sources are an optimal method for collision avoidance systems due to low cost and insensitivity to the weather. The relative position and real-time dynamic information between two GNSS users can be obtained from wireless communication in order to assess the safety situation for the avoidance of rear-end collisions. In current GNSS based rear-end collision avoidance systems, however, the accuracy of the real-time estimation of positioning and dynamic states can be affected by abrupt manoeuvers by the drivers [7-9]. Although a reliable algorithm for extracting the car-following status is therefore essential, the reliability and robustness of the GNSS fusion will also be critical for successful rear-end collision avoidance systems.

In this paper, a GNSS/compass fusion/lane information fusion, with an Adaptive Neurofuzzy Inference System (ANFIS) based Vehicle-to-Vehicle (V2V) rear-end collision avoidance system is developed. The estimation of real-time vehicle states is achieved using a Cubature Kalman Filter(CKF-) based algorithm. The different features extracted from the fusion results, that is, the Relative Distance, velocity, and heading between the leading vehicle and following vehicle, are used as input to the ANFIS for its automatic FIS membership functions and rules generation based on its learning algorithm. The car-following status is therefore predicted based on the ANFIS output. The contributions of the paper are summarized as follows:

(1) A newly designed CKF model for real-time vehicle status estimation

(2) A novel ANFIS-based car-following status decision algorithm with the advantage of early prediction warning and high detection accuracy

(3) Field experiments presented to demonstrate the successful application of the designed rear-end collision avoidance algorithm.

The rest of the paper is organized as follows. The design of the GNSS fusion-based rear-end collision avoidance system is presented in Section 2. In particular, the fusion of GNSS, compass, and lane information data for the estimation of

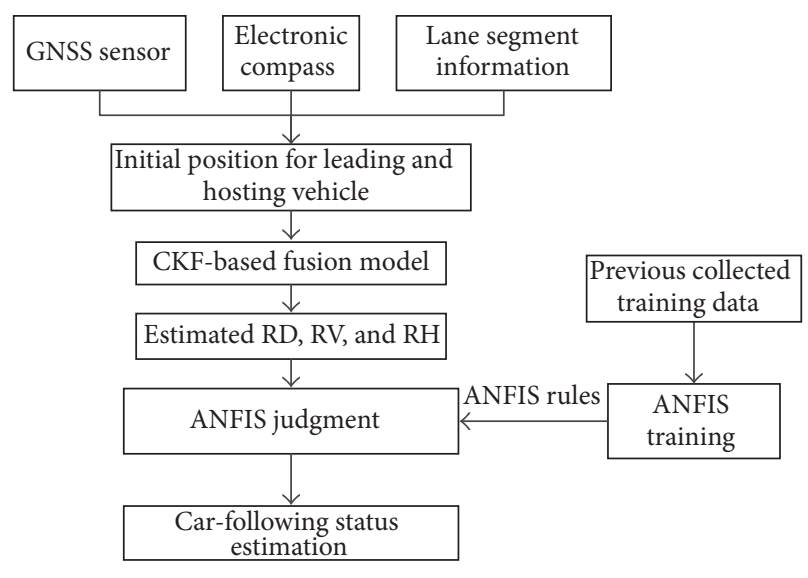

FIgURE 1: System overview.

vehicle states is presented in Section 2.2, and the ANFISbased rear-end collision status identification is presented in Section 2.3. The field test and the evaluation of the proposed algorithm are discussed in Section 3. The conclusion is in Section 4.

\section{GNSS Fusion-Based Rear-End Collision Avoidance System}

2.1. Method Overview. A flowchart of the CKF-based GNSS/compass fusion for Vehicle-to-Vehicle (V2V) rear-end collision avoidance system is illustrated in Figure 1. The two phases of the system are described as follows. The first phase is concerned with both vehicle position and dynamic states estimation. For each vehicle, one GNSS receiver and one electronic compass are mounted on the top of both vehicles for the collection of real-time positioning, velocity, and attitude data. The CKF-based fusion model is applied to the vehicles in order to estimate the vehicle states. The second phase involves the identification of the car-following status based on the estimated states from the first phase (position and dynamic states). Specifically, the Relative Distance (RD), Relative Velocity (RV), and Relative Heading (RH) calculated from the first phase are the input variables used for the ANFIS to predict the car-following status output. The fuzzy rules are extracted from previously collected labelled data based on ANFIS training. Finally, the ANFIS outputs for the testing data are calculated to evaluate the collision status.

\subsection{CKF-Based GNSS/Compass/Lane Information Fusion} Algorithm. The ability to locate and track the vehicles in space, velocity, and time is fundamental to predict collisions. It is therefore critical to choose an appropriate technology to determine the relative positioning and velocities of vehicles with sufficient accuracy and reliability to ensure high performance collision prediction. In order to improve the accuracy of GNSS for the collision avoidance application, GNSS/compass/lane information fusion is employed. Nonlinear filters, such as the Extended Kalman Filter (EKF), which linearizes the nonlinear system based on the 1storder Taylor series expansion, have been applied for GNSS 


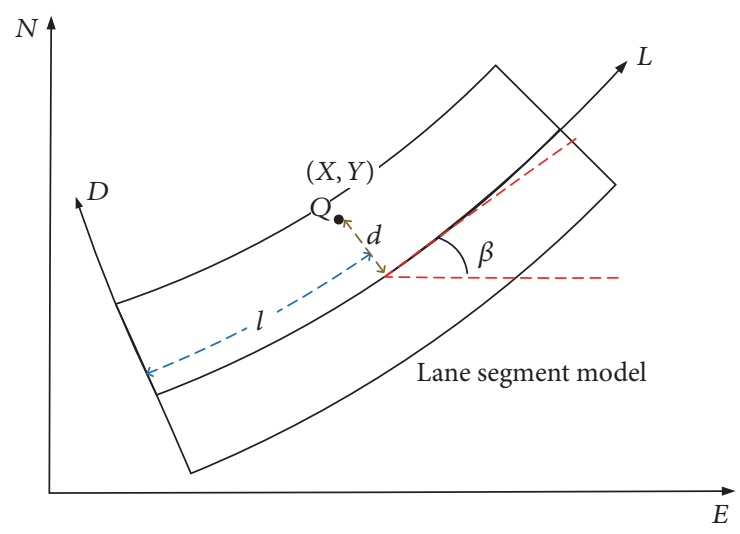

FIGURE 2: Lane segment model.

fusion for many years. Although EKF returns acceptable results in many ITS applications, it cannot however estimate vehicle manoeuvres accurately when sudden stops or turns occur, due to the shortcomings of Taylor linearization. In recent years, therefore, some improved algorithms have been developed based on EKF to improve the calculation accuracy and stability, such as UKF and CKF $[13,14]$. CKF, especially, is able to obtain good estimation accuracy with an acceptable computation complexity and has exhibited superior performance compared to UKF in many applications. The principle of CKF is to use the spherical-radial rule to get the basic cubature points and the opposite weights [14]. The mean and variance of the system state are propagated through a set of cubature points whose number is twice the dimension of the state vector. The cubature points and weights of CKF are therefore determined uniquely by the dimension of the state vector, which reduces the computation complexity.

The steps for the CKF-based GNSS/compass/lane information fusion are presented below.

(1) Definition of the State Vector. The state vector for a single vehicle is defined as (1) and the geometric relationships of the related parameters in the lane segment model are presented in Figure 2.

$$
(E N v \theta l d \beta)^{T} \text {, }
$$

where

$E, N$ are Easting and Northing coordinates (in meters) of the vehicle's geometric centre in a local coordinates system;

$L, D$ are longitudinal and lateral coordinates (in meters) of the lane segment;

$v$ is the heading velocity of the vehicle, output from the GNSS sensor;

$\theta$ is the heading of the vehicle, output from the compass sensor;

$l$ is the longitudinal displacement of the vehicle in lane segment coordinates;

$d$ is the lateral displacement of the vehicle in lane segment coordinates; $\beta$ is the tangent angle, which is the angle between the tangent line of the lane central line and the Eastingaxis coordinates.

(2) Spherical-Radial Rule. CKF uses the spherical-radial rule to find the cubature points and weights. The third-degree spherical-radial rule entails a total of $2 n$ cubature points when the dimension of the random variable equals $n$. The cubature points $\xi_{i}$ and their corresponding weights $\omega_{i}$ could be given as

$$
\begin{aligned}
\xi_{i} & =\sqrt{\frac{m}{2}}[1]_{i}, \\
\omega_{i} & =\frac{1}{m}, \quad(i=1,2, \ldots, m=2 n),
\end{aligned}
$$

where $m$ is the number of basic cubature points. In the equation, $[1]_{i}$ is denoted as the $i$ th member from the point group. For example, when $n=2$, the point group is $\left\{\left[\begin{array}{l}1 \\ 0\end{array}\right],\left[\begin{array}{l}0 \\ 1\end{array}\right],\left[\begin{array}{c}-1 \\ 0\end{array}\right],\left[\begin{array}{c}0 \\ -1\end{array}\right]\right\}$.

(3) Cubature Kalman Filter Calculation. Before the iteration of time update and measurement update steps for the CKF at each time-step, the cubature-point set $\left\{\xi_{i}, \omega_{i}\right\}$ should be computed based on (2). The detailed steps of the CKF are depicted as follows.

(i) Time update

(1) Assume at time $k$ that the posterior density function (PDF) is known. Factorize

$$
\mathbf{P}_{i, k-1 \mid k-1}=\mathbf{S}_{k-1 \mid k-1}\left(\mathbf{S}_{k-1 \mid k-1}\right)^{T},
$$

where the Cholesky decomposition is applied to factorize the covariance $\mathbf{P}_{k-1 \mid k-1}$, noted as $\mathbf{S}_{k-1 \mid k-1}=\operatorname{chol}\left(\mathbf{P}_{k-1 \mid k-1}\right)$.

(2) Evaluate the cubature points $(i=1,2, \ldots, m=$ $2 n)$ :

$$
\mathbf{X}_{i, k-1 \mid k-1}=\mathbf{S}_{k-1 \mid k-1} \xi_{i}+\widehat{\mathbf{x}}_{k-1 \mid k-1} .
$$

(3) Propagate cubature points $(i=1,2, \ldots, m)$ based on state-update function (5) so that the predicted state can be estimated by means of (6). The function $f(\cdot)$ is related to the vehicle motion model. The Constant Acceleration (CA) model is used here as it has been proved to provide a quick and reasonable estimation for the motion of vehicles [15].

$$
\begin{aligned}
\mathbf{X}_{\mathbf{i}, k \mid k-1}^{*} & =\mathbf{f}\left(\mathbf{X}_{\mathbf{i}, k-1 \mid k-1}\right), \\
\widehat{\mathbf{x}}_{k \mid k-1} & =\frac{\mathbf{1}}{\mathbf{m}} \sum_{\mathbf{i}=\mathbf{1}}^{\mathbf{m}} \mathbf{X}^{*}{ }_{i, k \mid k-1} .
\end{aligned}
$$

(4) Calculation of predicted error covariance:

$$
\mathbf{P}_{k \mid k-1}=\frac{1}{m} \sum_{i=1}^{m} \mathbf{X}_{i, k \mid k-1}^{*} \mathbf{X}_{i, k \mid k-1}^{* \mathbf{T}}-\widehat{\mathbf{x}}_{k \mid k-1} \widehat{\mathbf{x}}_{k \mid k-1}+\mathbf{Q}_{k-1} .
$$


(ii) Measurement update

(1) Factorize

$$
\mathbf{P}_{k \mid k-1}=\mathbf{S}_{k \mid k-1}\left(\mathbf{S}_{k \mid k-1}\right)^{T} .
$$

(2) Evaluate the cubature points $(i=1,2, \ldots, m)$ and the propagated cubature points:

$$
\begin{aligned}
& \mathbf{X}_{i, k \mid k-1}=\mathbf{S}_{k \mid k-1} \xi_{i}+\widehat{\mathbf{x}}_{k \mid k-1}, \\
& \mathbf{Z}_{i, k \mid k-1}=h\left(\mathbf{X}_{i, k \mid k-1}\right) .
\end{aligned}
$$

(3) Update the output vectors: calculate predicted measurement and the innovation covariance matrix according to (10) and (11), respectively:

$$
\begin{aligned}
\widehat{\mathbf{z}}_{k \mid k-1} & =\frac{1}{m} \sum_{i=1}^{1} \mathbf{Z}_{i, k \mid k-1}, \\
\mathbf{P}_{z z, k \mid k-1} & =\sum_{i=1}^{m} \mathbf{Z}_{i, k \mid k-1} \mathbf{Z}_{i, k \mid k-1}^{\mathrm{T}}-\widehat{\mathbf{z}}_{k \mid k-1} \widehat{\mathbf{z}}_{k \mid k-1}^{\mathrm{T}}+\mathbf{R}_{k} .
\end{aligned}
$$

(4) Calculate the cross covariance matrix and the cubature Kalman gain. The cross covariance matrix and the cubature Kalman gain vector are calculated according to (12) and (13), respectively:

$$
\begin{aligned}
\mathbf{P}_{x z, k \mid k-1} & =\frac{1}{m} \sum_{i=1}^{m} \mathbf{Z}_{i, k \mid k-1} \mathbf{Z}_{i, k \mid k-1}^{\mathbf{T}}-\widehat{\mathbf{x}}_{k \mid k-1} \widehat{\mathbf{z}}_{k \mid k-1}^{\mathbf{T}}, \\
\mathbf{W}_{\mathbf{k}} & =\mathbf{P}_{\mathbf{x z}, \mathbf{k} \mid \mathbf{k}-\mathbf{1}} \mathbf{P}_{\mathbf{z z}, \mathbf{k} \mid \mathbf{k}-\mathbf{1}}^{-\mathbf{1}} .
\end{aligned}
$$

(5) Update the state and the corresponding error covariance. Calculation of the estimated state and the covariance based on the generic Kalman Filter:

$$
\begin{aligned}
& \widehat{\mathbf{x}}_{k \mid k}=\widehat{\mathbf{x}}_{k \mid k-1}+\mathbf{W}_{k}\left(z_{k}-\widehat{\mathbf{z}}_{k \mid k-1}\right), \\
& \mathbf{P}_{k \mid k}=\mathbf{P}_{k \mid k-1}-\mathbf{W}_{k} \mathbf{P}_{z z, k \mid k-1} \mathbf{W}_{k}^{T} .
\end{aligned}
$$

The variables used in the CKF algorithm are illustrated as follows:

$\mathbf{S}_{\mathbf{k}}$ : the parameter factorized from covariance $\mathbf{P}_{\mathbf{k}}$ based on the Cholesky decomposition

$\widehat{\mathbf{x}}_{\mathbf{k}}$ : estimated state vector at step $k$

$\widehat{\mathbf{z}}_{\mathbf{k}}$ : estimated measurement vector at step $k$

$\mathbf{Z}_{\mathbf{k}}$ : measurement vector at step $k$

$\mathbf{X}^{*}{ }_{\mathbf{k}}$ : propagated cubature points at step $k$

$\mathbf{P}_{\mathbf{k}}$ : covariance matrix of the state vector at step $k$

$\mathbf{P}_{\mathbf{z z}, \mathbf{k}}$ : covariance matrix of the measurement vector at step $k$

$\mathbf{P}_{\mathbf{x z}, \mathbf{k}}$ : cross covariance matrix of the state vector and measurement vector at step $k$ $\boldsymbol{\xi}_{\mathbf{i}}$ : cubature points with $i$ th column of the matrix

$\omega_{\mathbf{i}}$ : weights for cubature points with $i$ th column of the matrix

$\mathbf{Q}_{\mathbf{k}}$ : covariance matrix of process noise at step $k$

$\mathbf{R}_{\mathbf{k}}$ : covariance matrix of measurement noise at step $k$

$\mathbf{W}_{\mathbf{k}}$ : cubature Kalman gain vector

2.3. ANFIS-Based Collision Avoidance System. Fuzzy Inference Systems (FIS) can be used to link nonlinear phenomena to relative variables based on fuzzy logic rules, since this is difficult to model using conventional mathematical models. In contrast to traditional binary logic theory, fuzzy logic variables define the true value of the system as partially true or false with a value ranging from 0 to 1 . With this advantage over traditional logic, FIS has been widely used for vehicle collision warning systems in recent years $[16,17]$. Two issues are essential for the performance of FIS-based collision avoidance systems. One is the method for transforming the experienced data for the rules training of the FIS, and the other is the effective tuning of the membership functions to increase the system performance, that is, the balance between the false alarm rate and the correct detection rate. The Adaptive Neurofuzzy Inference System (ANFIS) is able to adaptively extract the fuzzy rules from the experienced input data based on neural network training and apply the trained rules on the Sugeno type fuzzy based decision system and is therefore able to combine the traditional advantages of FIS (i.e., transparency and the application of expert knowledge within its structure) with the advantages of neural networks (i.e., their fast learning capability) [18]. In the designed rear-end collision detection system, the Relative Distance (RD), Relative Velocity (RV) and Relative Heading (RH) for the following and leading vehicles are defined as the input variables for the FIS. The resulting structure of the ANFISbased collision avoidance system therefore has five layers, as illustrated in Figure 3.

For a first-order Sugeno fuzzy model, a common rule is given below.

Rule 1. If $x$ is $A_{1}$ and $y$ is $B_{1}$ and $z$ is $C_{1}$, then

$$
f_{1}=p_{1} * x+q_{1} * y+r_{1} * z+s_{1},
$$

where the parameters defining $A_{1}, B_{1}$, and $C_{1}$ membership function, along with $p_{1}, q_{1}, r_{1}$, and $s_{1}$, are modified during the training. The description of each layer in ANFIS is as follows.

Layer 1. Assume every node $i$ in this layer is a square node with a node function

$$
O_{i}^{1}=\mu A_{i}(x),
$$

where $x$ is the input of node $i$ and $A_{i}$ is the linguistic label (e.g., small, medium, and large) with node $i . O_{i}^{1}$ is the membership function of $A_{i}$. In our case, the initial membership functions of the input variables are set as the Gaussian ones based on the character of the input information:

$$
\operatorname{Gaussian}(x ; \sigma, c)=e^{-(x-c)^{2} / 2 \sigma^{2}},
$$




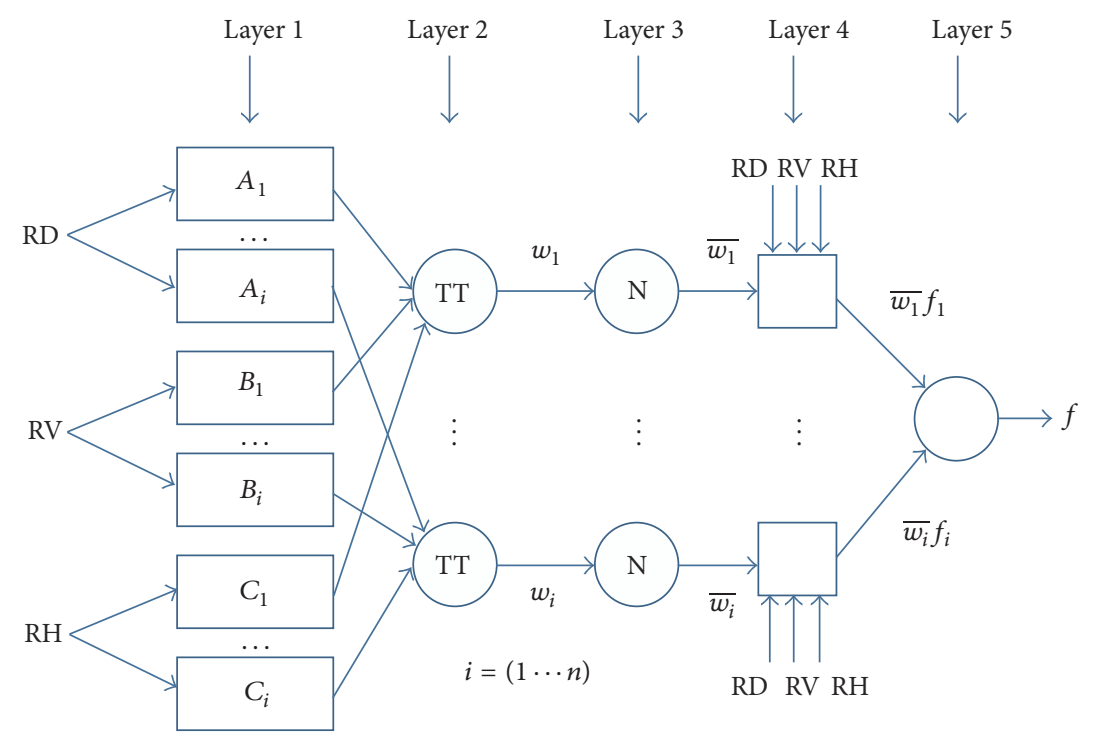

FIGURE 3: Structure of the ANFIS-based collision avoidance system.

where $c$ is the parameter to determine the centre of the membership function and $\sigma$ determines the width of the curve. The parameters in this layer are considered to be the premise parameters.

Layer 2. Each node in this layer calculates the firing strength of each rule via multiplication. In our case, we use an AND $T$-norm operator here, given by

$$
O_{i}^{2}=w_{i}=\mu A_{i}(x) * \mu B_{i}(y) * \mu C_{i}(z), \quad i=1,2,3 .
$$

Layer 3. The $i$ th node of this layer calculates the ratio of the $i$ th rule's firing strength to the sum of the firing strengths of all the rules.

$$
\mathrm{O}_{i}^{3}=w_{i}=\frac{w_{i}}{w_{1}+w_{2}+w_{3}}, \quad i=1,2,3 .
$$

Layer 4 . The multiplication for input from layer 3 and layer 1 is implemented, given by

$$
O_{i}^{4}=\bar{w}_{i} f_{i}=\overline{w_{i}}\left(p_{i} * x+q_{i} * y+r_{i} * z+s_{i}\right),
$$

where $\overline{w_{i}}$ is the output of layer 3 and $\left\{p_{i} q_{i} r_{i}\right\}$ is the parameter set. Parameters in this layer are called consequent parameters.

Layer 5. It computes the overall outputs as the summation of all incoming signals.

$$
\sum_{i} \overline{w_{i}} f_{i}=\frac{\sum_{i} w_{i} * f_{i}}{\sum_{i} w_{i}}
$$

Subtractive clustering is applied for the initial FIS design to improve the calculation speed. In addition, during the learning process, the premise parameters in layer 1 and the consequent parameters in layer 4 are tuned until the desired response of the FIS is achieved. A hybrid learning algorithm combining the Least Square Method (LSM) and the backpropagation (BP) algorithm is employed for this training.

Once the FIS rules are obtained after the training, they can be used for any input variables in order to get the corresponding output values. For example, if we apply the extracted FIS rules on the set of input Relative Distance (RD), Relative Velocity (RV), and Relative Heading (RH) for the following and leading vehicles, the corresponding output value can be predicted. In this paper, we define the warning status (labelled as " 1 ") and normal status (labelled as "0") for the output value classification. The predicted values from the ANFIS will be rounded to the integer " 0 " or " 1 " for the classification. The details will be discussed in the next section.

\section{Field Test and Analysis}

The performance of the designed CKF-based GNSS/compass/lane information fusion for the avoidance of vehicle rear-end collisions will be discussed in this section. The experiment setup and data collection will be introduced in Section 3.1; the performance assessment of the CKFbased fusion algorithm, and the fusion, will be discussed in Section 3.2; the performance of the GNSS fusion and ANFIS-based car-following status identification system will be discussed in Section 3.3.

3.1. Experiment Setup and Data Collection. The car-following data was collected near Lincheng Industrial Park, Zhoushan City, China. The data used in the experiment includes the training data and testing data. The training data was collected in advance with their danger status recorded and labelled. In order to ensure the safety of the experiment, a simulated very close car-following situation was used throughout the whole experiment instead of real collisions. These data were collected and recorded based on the high 
TABLE 1: The comparison of the navigation performance of the leading and following vehicles.

\begin{tabular}{|c|c|c|c|}
\hline \multirow{2}{*}{ Positioning method } & \multicolumn{3}{|c|}{ Following vehicle performance (Buick) } \\
\hline & Positon RMSE (m) & Velocity RMSE $(\mathrm{m} / \mathrm{s})$ & Availability \\
\hline RTK GNSS only & 0.4473 & 2.8692 & $97.58 \%$ \\
\hline CKF-based fusion results & 0.3025 & 2.1524 & $100 \%$ \\
\hline \multirow{2}{*}{ Positioning method } & \multicolumn{3}{|c|}{ Leading vehicle performance (Nissan) } \\
\hline & Positon RMSE (m) & Velocity RMSE $(\mathrm{m} / \mathrm{s})$ & Availability \\
\hline RTK GNSS only & 0.3609 & 2.682 & $98.94 \%$ \\
\hline CKF-based fusion results & 0.2056 & 2.1027 & $100 \%$ \\
\hline
\end{tabular}

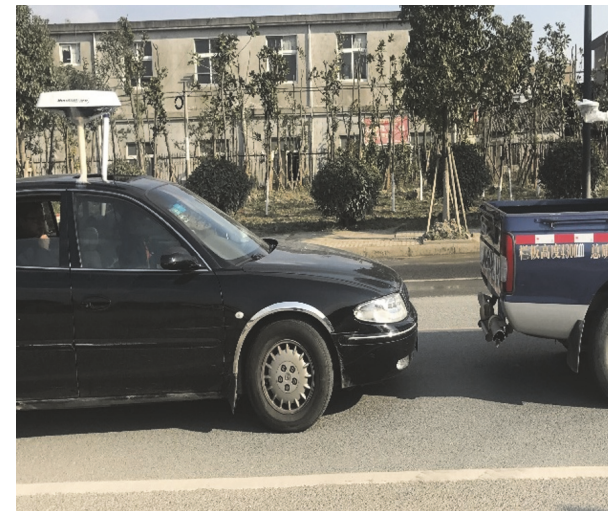

(a)

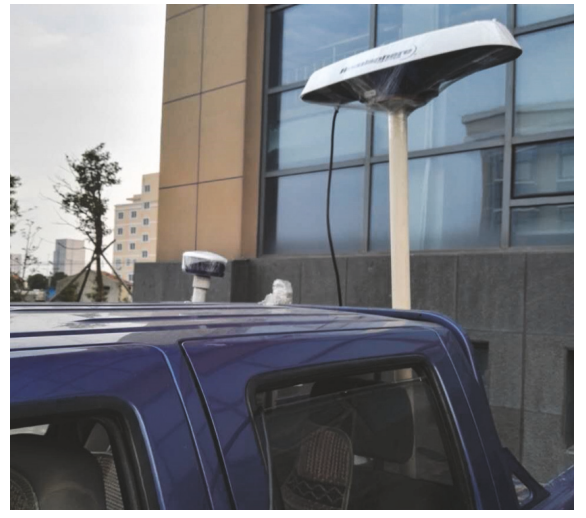

(b)

FIGURE 4: Demonstration of a rear-end collision in the test (a) and the onboard equipment (b).

grade GNSS/Inertial Navigation System (INS) integrated sensors. Different manoeuvres were performed manually and recorded. For the dangerous driving behaviours, the driver of the following vehicle conducted aggressive manoeuvres, including abrupt acceleration and deceleration with different velocities and headings so that the following vehicle closed rapidly with the leading vehicle. For the normal data, we just drove smoothly and maintained a distance of more than $5 \mathrm{~m}$ between the two cars (here the distance used is the distance between two antennas on both cars). We tried our best to simulate driving situations that would represent different types of dangerous status in real driving. The testing rearend collision data were captured from 07:15:00 to 07:26:00 in Universal Coordinated Time (UTC) with total five times of the simulated collision sessions. During the test, the Buick was assigned as the following vehicle and the Nissan was assigned as the leading vehicle. The test vehicles and onboard sensors are shown in Figure 4.

For both vehicles, two types of data were collected and used in the field test: (1) the reference data, which is the postprocessed data output from the integrated GNSS/INS with the video recorded and labelled collision situations; (2) the Real-Time Kinematic (RTK) GNSS and compass data for both vehicles in the test sessions. The frequency of the collected data for both vehicles is $10 \mathrm{~Hz}$.

In order to obtain the real-time lateral displacement and curvature angle of the vehicle in the related lane segment, the coordinates of the lane's central line for the experiment area were collected in advance by a vehicle with a high grade integrated sensor. This data was then postprocessed to be recognized as the position of the lane's central line. The lateral displacement of the vehicle was calculated by finding the two measurement points on the central line that were closest to the vehicle and then calculating the perpendicular distance from the vehicle to the line segment containing these two points.

\subsection{Analysis of the CKF-Based GNSS/Compass/Lane Infor-} mation Fusion. This section discusses the CKF-based GNSS/ compass/lane information fusion algorithm for the estimation of the positioning and dynamic parameters for both the leading and following vehicles. Table 1 shows that the accuracy and availability improved for both the following and leading vehicles compared to the positioning results from RTK GNSS only. Figure 5 is an example of the CKF-based fusion results for the Nissan vehicle (i.e., the leading vehicle). It shows that the fusion algorithm has not only bridged the gaps in the GNSS positioning results, but also improved the accuracy and availability of the vehicle navigation performance. In addition, the velocity and heading estimations have also been improved based on the fusion algorithm, which will be essential for the identification of the car-following status.

\subsection{Analysis of Fusion and ANFIS-Based Car-Following Status} Identification Algorithm. The training data used for ANFIS rules extraction contained a total of 18151 samples, including 865 samples considered as having a collision warning status (labelled as "1") and 17286 samples with normal status 

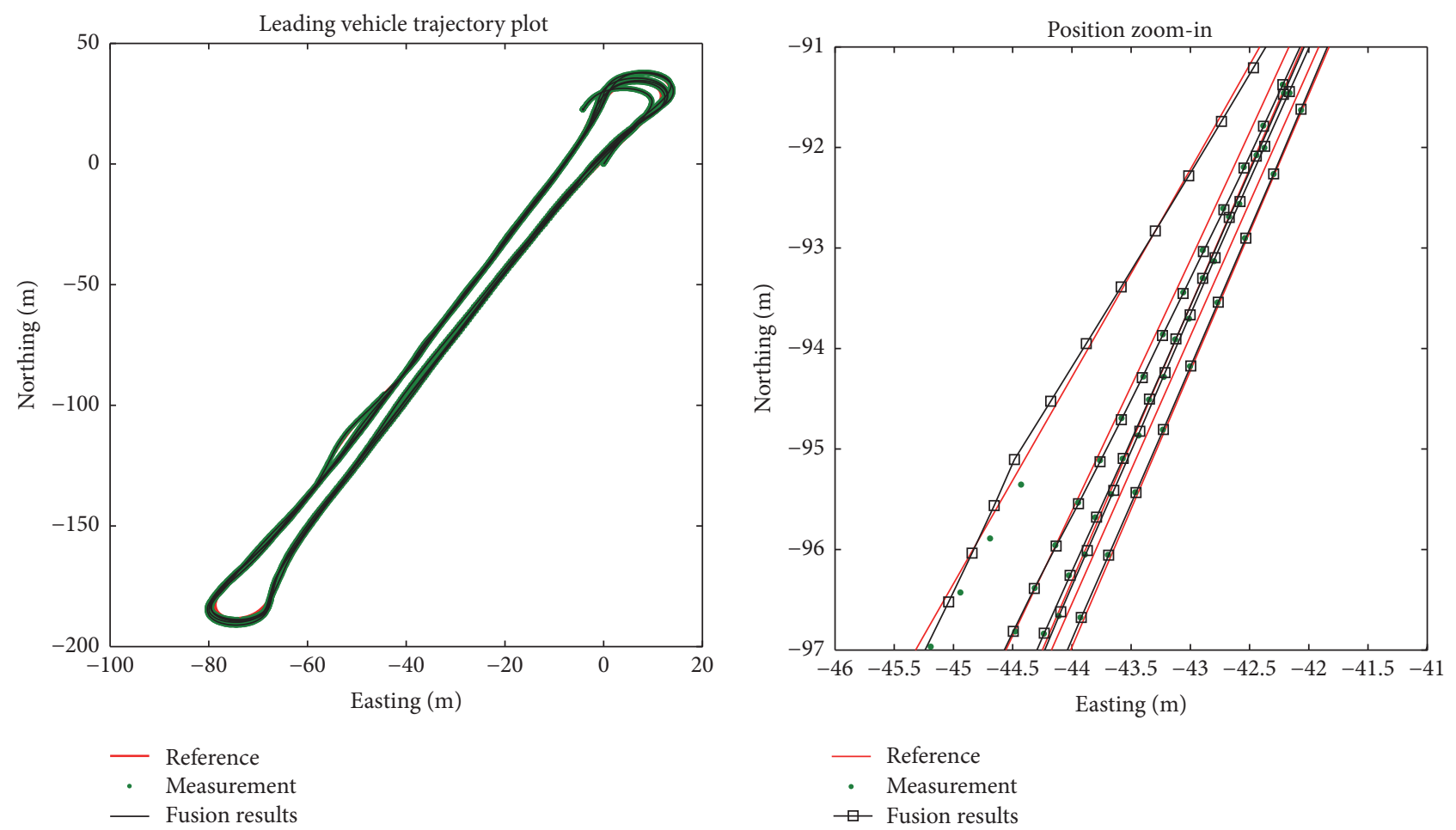

Figure 5: The trajectory of the leading vehicle and the comparison between the fusion results and the measurements with respect to the reference.

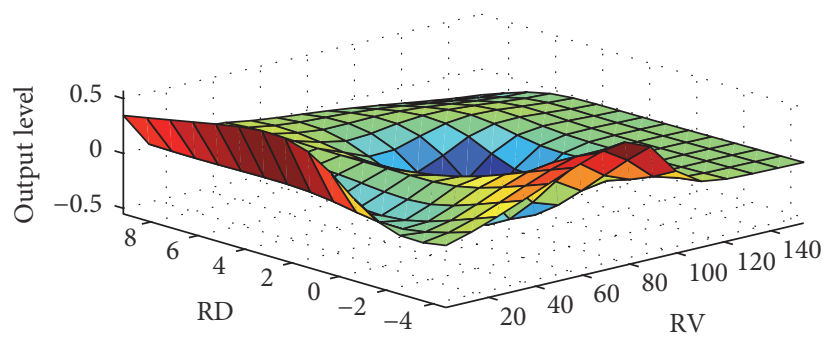

FIGURE 6: Surface view of the trained rules for RV and RD and the corresponding output level.

(labelled as “0”). The testing data includes 1809 samples with 76 with collision warning and 1733 normal ones. Based on the adaptive training of data for 100 steps, the rules for the input $\mathrm{RV}, \mathrm{RD}, \mathrm{RH}$, and the output status can be established; see Figure 6 as an example of the RV, RD, and the corresponding output level extracted. It is indicated that every pair of RV and $\mathrm{RD}$ has a corresponding output level value. The membership functions for the $\mathrm{RV}, \mathrm{RD}$, and $\mathrm{RH}$ before the training and after the training are shown in Figure 7. The levels of the initial membership functions of the input variables have been preliminary defined based on the Gaussian function. The premise membership function has been adaptively changed after the training, especially for the input variable $\mathrm{RH}$. It is indicated in the variable $\mathrm{RH}$ that the initial levels of the membership function (e.g., levels 1, 2, and 4) are very close to each other, but they become more separated after the neural network training.
The comparison between the fusion results with the ANFIS predicted output level, and the reference output level, are displayed in Figure 8. It is clear that the collision warning status can be identified with a high success rate. The confusion matrix of the identification results using reference data with ANFIS and GNSS/compass/lane information fusion with ANFIS is listed and compared in Table 2. The predicted values are rounded to the integer "0" or "1." Detection accuracy is calculated as the ratio (in percentage) of the number of correctly detected activities to the number of total known activities and false alarm rate as the ratio of the number of false positive activities ( 0 but detected as 1 ) to the total number of detected faults. As calculated from the table, the accuracy of the GNSS fusion with ANFIS predicted results is $99.61 \%$, while the false alarm rate is $5.26 \%$. The designed algorithm therefore exhibits a very close performance to the reference data, which has an accuracy of $99.78 \%$ and false 
TABLE 2: Confusion matrix of the identification results.

\begin{tabular}{|c|c|c|c|c|c|c|}
\hline & \multicolumn{3}{|c|}{ GNSS fusion with ANFIS predicted results } & \multicolumn{3}{|c|}{ Reference with ANFIS predicted results } \\
\hline \multirow{3}{*}{ Labelled result } & & 0 & 1 & & 0 & 1 \\
\hline & 0 & 1730 & 4 & 0 & 1731 & 3 \\
\hline & 1 & 3 & 72 & 1 & 1 & 74 \\
\hline
\end{tabular}
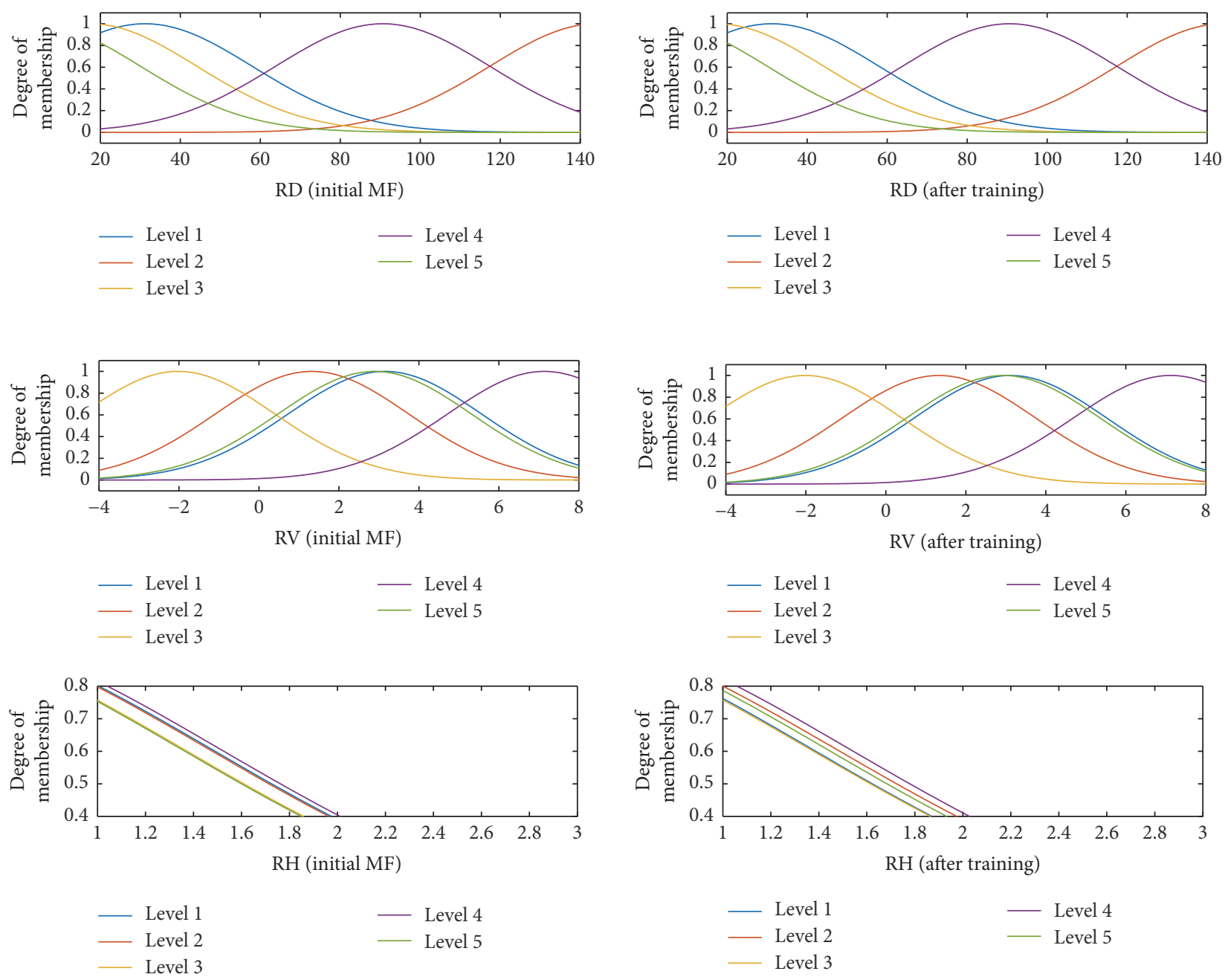

FIgURE 7: Membership function before and after training with ANFIS.

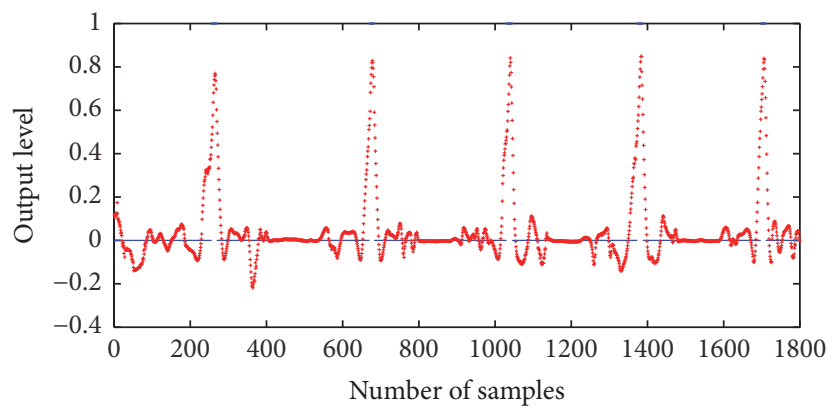

FIGURE 8: Comparison between the fusion results with ANFIS predicted output level and the reference output level. 
TABLE 3: The confusion matrix of the identification results.

\begin{tabular}{lccc}
\hline Performance & Proposed algorithm & Fuzzy logic based algorithm in [10] & Distance-based algorithm in [8] \\
\hline Accuracy & $99.61 \%$ & $98.34 \%$ & $97.18 \%$ \\
False alarm rate & $5.26 \%$ & $26.32 \%$ & $39.29 \%$ \\
\hline
\end{tabular}

alarm rate of $3.9 \%$. It is therefore clear that the low cost sensor fusion and ANFIS-based car-following status identification algorithm could be effectively used for collision avoidance systems.

In this section we compare the proposed algorithm with the most commonly used state-of-the-art algorithm from relevant literature. According to our literature review, although research to date has explored a number of aspects of rear-end collision detection, the assumptions and test data used for such collision detection are different. Nonetheless, some of their methodologies can still be adopted to design rear-end collision avoidance detection by using the field test data in this paper. These typical methods include the traditional fuzzy logic based algorithm with the input of Time to Collision (TTC) and Time Gap (TG) based collision avoidance system in [10] and the commonly used V2V distancebased collision avoidance algorithm in [8]. The performances of the collision detection results for these systems in terms of accuracy and false alarm rate are illustrated in Table 3.

It can be seen that the proposed algorithm outperforms its competitors. Although the accuracy for the proposed algorithm (i.e., 99.61\%) is only slightly higher than the algorithm in [10] (i.e., 98.34\%) and the distance-based algorithm in [8] (i.e., 97.18\%), the proposed algorithm exhibited the lowest false alarm rate of $5.26 \%$, compared to $26.32 \%$ and $39.29 \%$ for the fuzzy logic based algorithm in [10] and distance-based algorithm in [8]. The possible reasons for the high false alarm rate of these two state-of-the-art algorithms could be that the fuzzy logic based algorithm in [10] only uses a traditional fuzzy logic algorithm, which defines the rules manually without tuning the membership function to the optimal value, therefore, resulting in a high false alarm rate. The distance-based algorithm in [8], meanwhile, only considers the simple distance-based factor and not the velocity and heading, which will also be important factors for rear-end collision prediction, thus again leading to a high false alarm rate.

\section{Conclusion}

This paper presents a novel rear-end collision detection algorithm by combining CKF-based GNSS/compass/lane segment fusion with an ANFIS decision system. The field test has demonstrated the practicality of this approach using cost-effective sensors and relative map information. It is shown that the proposed algorithm has not only improved the positioning accuracy and availability of the vehicle navigation performance, but also provides solid collision avoidance detection with high detection accuracy (i.e., 99.61\%) and a low false alarm rate (i.e., $5.26 \%$ ) at a $10 \mathrm{~Hz}$ output rate. In the future, more indicators will be developed to evaluate the designed algorithm and comparisons will be carried out between the designed algorithm and the other advanced algorithms using more scenarios.

\section{Conflicts of Interest}

The authors declare that they have no conflicts of interest.

\section{Acknowledgments}

This work is partially supported by the National Natural Science Foundation of China (no. 41704022, no. 61601228), National Natural Science Foundation of Jiangsu Province (nos. BK20170780, BK20161021), Fundamental Research Funds for the Central Universities (no. NJ20160015, no. NS2017043), and the Natural Science Foundation of Jiangsu Higher Education Institution (15KJB510016). The authors express thanks to the staff in Zhejiang Zhongyu Communication Co., Ltd, for their support in the field test.

\section{References}

[1] The Effect of High-Visibility Enforcement on Driver Compliance with Pedestrian Right-of-Way Laws: 4-Year Follow-up. Traffic technology transfer series, National Highway Traffic Safety Administration, 2017.

[2] N. Maziar and B. Jing, "Rear-end collision: causes and avoidance techniques," in Wireless Vehicular Networks for Car Collision Avoidance, pp. 99-119, 2013.

[3] H. Araki, K. Yamada, Y. Hiroshima, and T. Ito, "Development of rear-end collision avoidance system," in Proceedings of the 1996 IEEE Intelligent Vehicles Symposium, pp. 224-229, September 1996.

[4] H. Araki, K. Yamada, Y. Hiroshima, and T. Ito, "Development of rear-end collision avoidance system," JSAE Review, vol. 18, no. 3, pp. 314-316, 1997.

[5] D. H. Tsai, "Vehicle safety and collision warning system," in Proceedings of 5th Asia-Pacific ITS Forum, Seoul, South Korea, 2002.

[6] J. Ueki, S. Tasaka, Y. Hatta, and H. Okada, "Vehicular-Collision Avoidance Support System (VCASS) by inter-vehicle communications for advanced ITS," IEICE Transactions on Fundamentals of Electronics, Communications and Computer Sciences, vol. E88-A, no. 7, pp. 1816-1823, 2005.

[7] J. Huang and H.-S. Tan, "Design and implementation of a cooperative collision warning system," in Proceedings of the IEEE Intelligent Transportation Systems Conference, pp. 10171022, 2006.

[8] R. Ong and G. Lachapelle, "Use of GNSS for vehicle-pedestrian and vehicle-cyclist crash avoidance," International Journal of Vehicle Safety, vol. 5, no. 2, pp. 137-155, 2011.

[9] R. Toledo-Moreo and M. A. Zamora-Izquierdo, "Collision avoidance support in roads with lateral and longitudinal maneuver prediction by fusing GPS/IMU and digital maps," 
Transportation Research Part C: Emerging Technologies, vol. 18, no. 4, pp. 611-625, 2010.

[10] V. Milanés, J. Pérez, J. Godoy, and E. Onieva, "A fuzzy aid rearend collision warning/avoidance system," Expert Systems with Applications, vol. 39, no. 10, pp. 9097-9107, 2012.

[11] L. Ujjainiya and M. K. Chakravarthi, "Raspberry-pi based cost effective vehicle collision avoidance system using image processing," ARPN Journal of Engineering and Applied Sciences, vol. 10, no. 7, pp. 3001-3005, 2015.

[12] O. Alpar and R. Stojic, "Intelligent collision warning using license plate segmentation," Journal of Intelligent Transportation Systems: Technology, Planning, and Operations, vol. 20, no. 6, pp. 487-499, 2016.

[13] E. A. Wan and D. M. R. Van, "The unscented Kalman filter for nonlinear estimation," in Proceedings of the IEEE Adaptive Systems for Signal Processing, Communications, and Control Symposium (AS-SPCC '00), pp. 153-158, Lake Louise, Canada, October 2000.

[14] I. Arasaratnam and S. Haykin, "Cubature Kalman filters," Institute of Electrical and Electronics Engineers Transactions on Automatic Control, vol. 54, no. 6, pp. 1254-1269, 2009.

[15] R. Sun, W. Y. Ochieng, and S. Feng, "An integrated solution for lane level irregular driving detection on highways," Transportation Research Part C: Emerging Technologies, vol. 56, pp. 61-79, 2015.

[16] F. Cheng, D. Zhu, and Z. Xu, "The study of vehicle's anticollision early warning system based on fuzzy control," in Proceedings of the 2010 International Conference on Computer, Mechatronics, Control and Electronic Engineering, CMCE 2010, pp. 275-277, China, August 2010.

[17] M.-H. Kim, S. Lee, K.-N. Ha, and K.-C. Lee, "Implementation of a fuzzy-inferencebased, low-speed, close-range collisionwarning system for urban areas," Proceedings of the Institution of Mechanical Engineers, Part D: Journal of Automobile Engineering, vol. 227, no. 2, pp. 234-245, 2013.

[18] J. S. R. Jang, "ANFIS: adaptive-network-based fuzzy inference system," IEEE Transactions on Systems, Man, and Cybernetics, vol. 23, no. 3, pp. 665-685, 1993. 


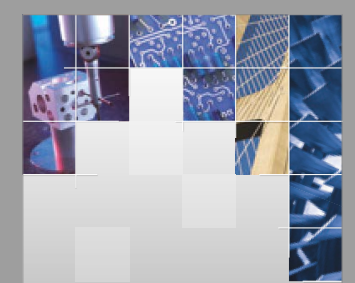

\section{Enfincering}
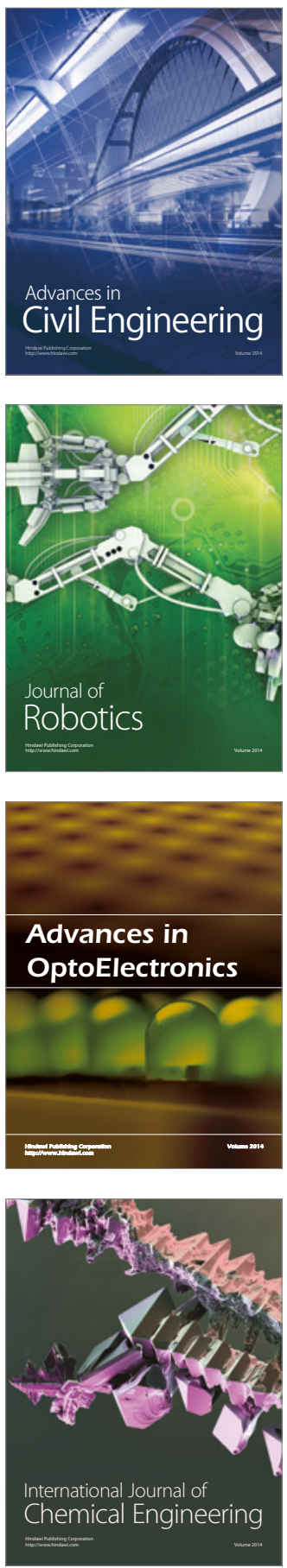

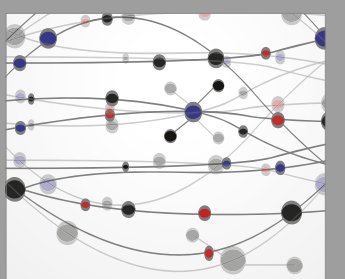

The Scientific World Journal

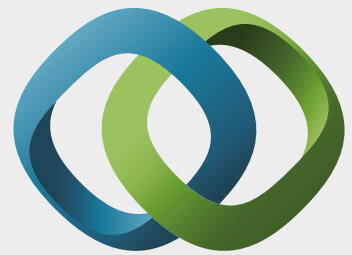

\section{Hindawi}

Submit your manuscripts at

https://www.hindawi.com
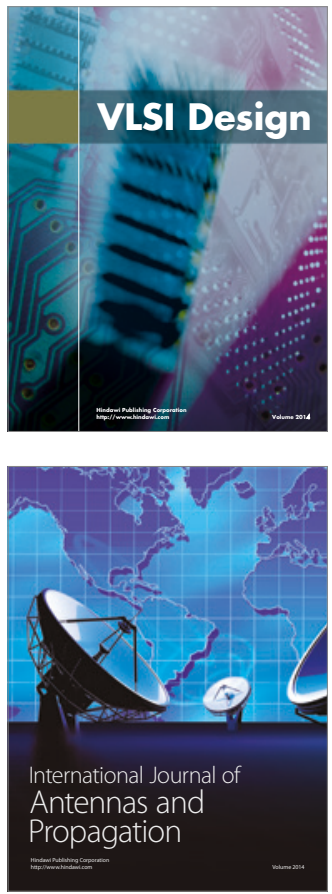

\section{Rotating}

Machinery
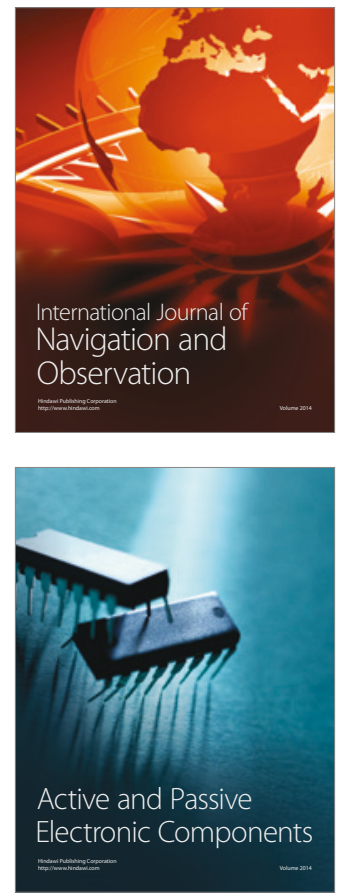
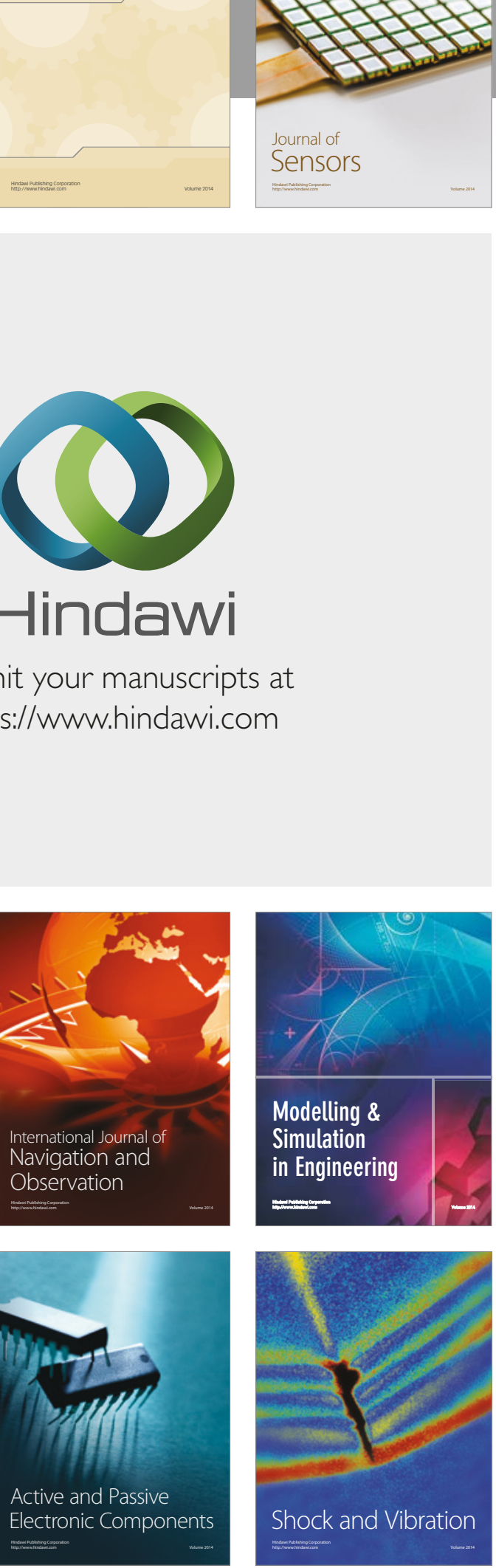
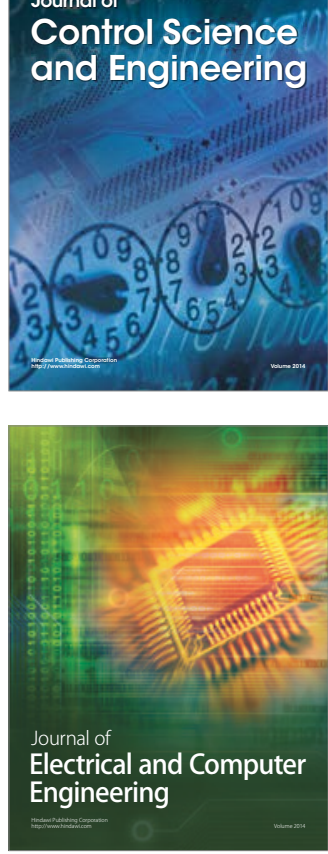

Distributed

Journal of

Control Science

and Engineering
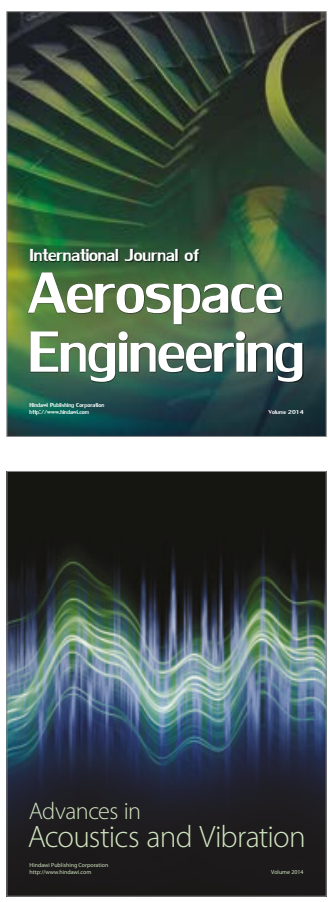

Sensor Networks 